\title{
El Derecho a la Educación en el Nivel Medio Superior en México
}

\section{The Right to Education in Higher Secondary Level in Mexico}

\author{
Ma Mercedes Ruiz Muñoz * \\ Alejandra Luna Guzmán \\ Universidad Iberoamericana
}

\begin{abstract}
Este trabajo se inscribe en un proyecto de investigación en marcha acerca del derecho a la educación y la justicia en América Latina. En el caso de México, el Estado tiene la obligación de garantizar la educación básica y media superior. Esta última, es considerada un derecho a lograrse de manera paulatina y creciente para el ciclo escolar 2021-2022. A la fecha de hoy, los avances son muy precarios y lentos y se enfrenta a enormes retos en materia de política educativa. A partir del modelo de las “4 As" de Tomasevski (2004), se analiza la situación de la educación media superior en México y se identifica que la asequibilidad es la dimensión que enfrenta mayores retos ya que aún se observa un déficit en materia de cobertura y distribución equitativa. Asimismo, el análisis de la accesibilidad, la aceptabilidad y la adaptabilidad invita a una reflexión más profunda sobre las orientaciones y los objetivos de la política educativa actual. Se concluye que la educación media superior es aún un derecho inalcanzable, lo que posibilita la emergencia de sujetos de derecho que expresan capacidad de agencia para su exigibilidad. Además, se requiere de una ciudadanía que acompañe la defensa de los derechos humanos, entre ellos el educativo. Tal como lo señala Latapí (2009) el derecho a la educación es considerado un derecho llave dado que abre la posibilidad de exigir otros derechos.
\end{abstract}

Descriptores: Derecho a la educación, Acceso a la educación, Política educacional, Reforma de la educación, Derechos humanos.

This work is part of an ongoing research project on the right to education and justice in Latin America. In the case of Mexico, the State has the obligation to guarantee basic and upper secondary education. The latter is considered a right to be achieved gradually and increasingly for the school year 2021-2022. To date, progress is very precarious and slow and faces enormous challenges in the field of educational policy. From the perspective of Tomasevski's (2004) model of the "4 As", the situation of higher education in Mexico is analyzed and it is identified that availability is the dimension that faces major challenges since there is still a deficit in coverage and equitable distribution. Likewise, the analysis of accessibility, acceptability and adaptability invites to a deeper reflection on the orientations and objectives of the current educational policy. It is concluded that upper secondary education is still an unattainable right, which makes possible the emergence of subjects of rights who express agency capability for its enforceability. In addition, citizenship is required to accompany the defense of human rights, including right to education. As Latapí (2009) highlights, the right to education is considered a key right since it opens the possibility of demanding other rights.

Keywords: Right to education, Access to education, Educational policy, Educational reform, Human rights. 


\section{A manera de introducción}

La educación se encuentra en un impasse entre el derecho y la política educativa. De acuerdo al Informe Mundial de Educación 2016, la universalización de la educación media superior en México, se logrará en el 2100 (UNESCO, 2016). En ese sentido, el derecho a la educación ha exigido políticas públicas y la política educativa ha acogido el derecho a la educación como su bandera. Los resultados, de cualquier manera, siguen siendo reprobables, baste mirar a las personas que están fuera del sistema educativo mexicano y las que ingresan y permanecen no logran los aprendizajes básicos.

Las distintas agencias internacionales han fijado su posicionamiento sobre el derecho a la educación. En el ámbito jurídico internacional, este derecho goza de reconocimiento por parte de los Estados miembro que firman y ratifican varios de estos instrumentos como el Pacto internacional de derechos económicos, sociales y culturales (Ruiz, 2012). Sin embargo, por tratarse de un derecho social o de segunda generación, se encuentra en riesgo de "adquirir un mero valor simbólico o político, pero poca virtualidad jurídica" (Didier, 2012, p. 82)

El derecho a la educación es conocido como un derecho humano progresivo o derecho llave (key right), es además un derecho que potencia la defensa de otros derechos humanos (Latapí, 2009). De acuerdo a la Declaración de los Derechos Humanos (1948), todos tenemos derecho a la educación gratuita y obligatoria, por lo menos la educación fundamental, hoy intitulada educación básica (preescolar, primaria y secundaria).

De acuerdo a Tomasevski, (2004, pp. 349-351), ex relatora del derecho a la educación por parte de la UNESCO, plantea cuatro características a considerar para el cumplimiento de este derecho, que, por sus iniciales, se identifican como el modelo de las “4 As". De esta manera, para asegurar el derecho a la educación con base en este esquema, se deben tomar en cuenta la asequibilidad, la accesibilidad, la aceptabilidad y la adaptabilidad de la educación. Para la autora, la asequibilidad se relaciona con la libertad de y en la educación, comprende la educación gratuita y obligatoria y el respeto a la diversidad de las minorías. La accesibilidad se refiere a la progresividad con que el derecho a la educación debe ir facilitando una educación obligatoria gratuita e inclusiva cuanto antes y en la postobligatoria en la medida de lo posible. La aceptabilidad engloba conjuntos de criterios relacionados con la calidad y la calidez de la educación, así como las cualidades profesionales de los maestros. La adaptabilidad se refiere a la capacidad de adecuar la educación al contexto sociocultural de las escuelas, los niños y los jóvenes, así como a la promoción de los derechos humanos a través de la educación. Estas dimensiones que propone Tomasevski (2004) se tomarán como base para el análisis de la situación del derecho a la educación en nivel medio superior en México.

Este artículo está organizado en tres apartados. El primero se refiere a los antecedentes de la investigación de la problemática planteada, a la estructura y las principales características de este nivel educativo. En el segundo apartado, se vinculan las obligaciones que tiene el Estado para garantizar la asequibilidad, la accesibilidad, la aceptabilidad y la adaptabilidad de la educación con los distintos indicadores y resultados educativos. Por último, se plantean las conclusiones del análisis y se invita a hacer una reflexión más profunda sobre las orientaciones y los objetivos de la política educativa actual. 


\section{La educación media superior en el contexto mexicano}

La educación a nivel medio superior en México ha atravesado por importantes cambios en los últimos años, en especial en lo relativo a la obligatoriedad y la gratuidad. Las distintas transformaciones en la educación básica permean a este nivel. Por ejemplo, uno de los aspectos más determinantes en estos cambios fue la firma del Acuerdo Nacional para la Modernización de la Educación Básica (ANMEB) en 1992, el cual, aunque orientado a la educación básica, de acuerdo a Loyo (2010, p. 40) definió "cimientos distintos para las políticas educativas en México (...) en un intento por conjugar innovación educativa con gobernabilidad para enfrentar los enormes retos de calidad y equidad educativa en el país”. Otro aspecto clave fue la entrada de México a la Organización para la Cooperación y el Desarrollo Económico (OCDE) en 1994, la cual implicó la participación del sistema educativo mexicano en el Programa Internacional para la Evaluación de Estudiantes (PISA, por sus siglas en inglés) que se realizó por primera vez en el año 2000 y marcó un parteaguas en la evaluación de la educación en México. Ambos aspectos echaron a andar un engranaje que dejó al descubierto la innegable necesidad de una profunda reforma en el sistema educativo, el ANMEB culminando en la promulgación de la Ley General de Educación en julio de 1993 y los resultados del Informe PISA ejerciendo la presión necesaria hasta la inclusión del derecho a la educación de calidad en la Constitución casi diez años después, el 23 de febrero de 2013.

En paralelo a estos cambios, también se fueron dando otros más profundos que tocaban la educación media superior en sus aspectos más estructurales. En principio, durante el gobierno de Vicente Fox, en 2005, se creó la Subsecretaría de Educación Media Superior. $\mathrm{Al}$ inicio de la administración de Felipe Calderón, el 9 de mayo de 2007 se anunció la puesta en marcha de la Reforma Integral de la Educación Media Superior (RIEMS) a fin de atender la demanda, calidad, equidad y pertinencia del nivel, así como la necesidad de imprimirle una identidad propia (Hamui y Villa, 2010). Nace así el Sistema Nacional de Bachillerato, que comparte un Marco Curricular Común (MCC) para todas las modalidades y subsistemas de la educación media superior. El MCC se compone de tres tipos de competencias: genéricas, disciplinares y profesionales con el objetivo de dotar a los egresados de igualdad de oportunidades. Al final del sexenio de Felipe Calderón, se realizaron algunas modificaciones al artículo $3^{\circ}$ Constitucional a través del artículo Segundo Transitorio, con las cuales se determinaba la obligatoriedad de la educación media superior de manera escalonada, iniciando a partir del ciclo escolar 2012-2013 y "se cumpliría hasta el ciclo 2021-2022, cuando debería alcanzarse la cobertura total” (Andere, 2013, p. 86). Sin embargo, con la reforma al artículo $3^{\circ}$ Constitucional de 2013, la obligatoriedad de dicho nivel se dio de facto y se vio reflejada en la Ley General de Educación el 10 de junio de ese mismo año.

De esta forma, la estructura de la educación media superior comprende los estudios posteriores a la secundaria, el denominado bachillerato, así como los equivalentes a éste y la educación profesional que no requiere estudios de bachillerato o sus equivalentes (figura 1) (SEMS, 2013a). Además, aunque no forman parte de su estructura, se encuentran bajo la coordinación de la Subsecretaría de Educación Media Superior el Colegio de Bachilleres (Colbach), el Colegio Nacional de Educación Profesional Técnica (Conalep), el Centro de Enseñanza Técnica Industrial (CETI) y el Fideicomiso de los Sistemas Normalizados de Competencia Laboral y de Certificación de Competencia Laboral (Conocer) (SEMS, 2013a). 


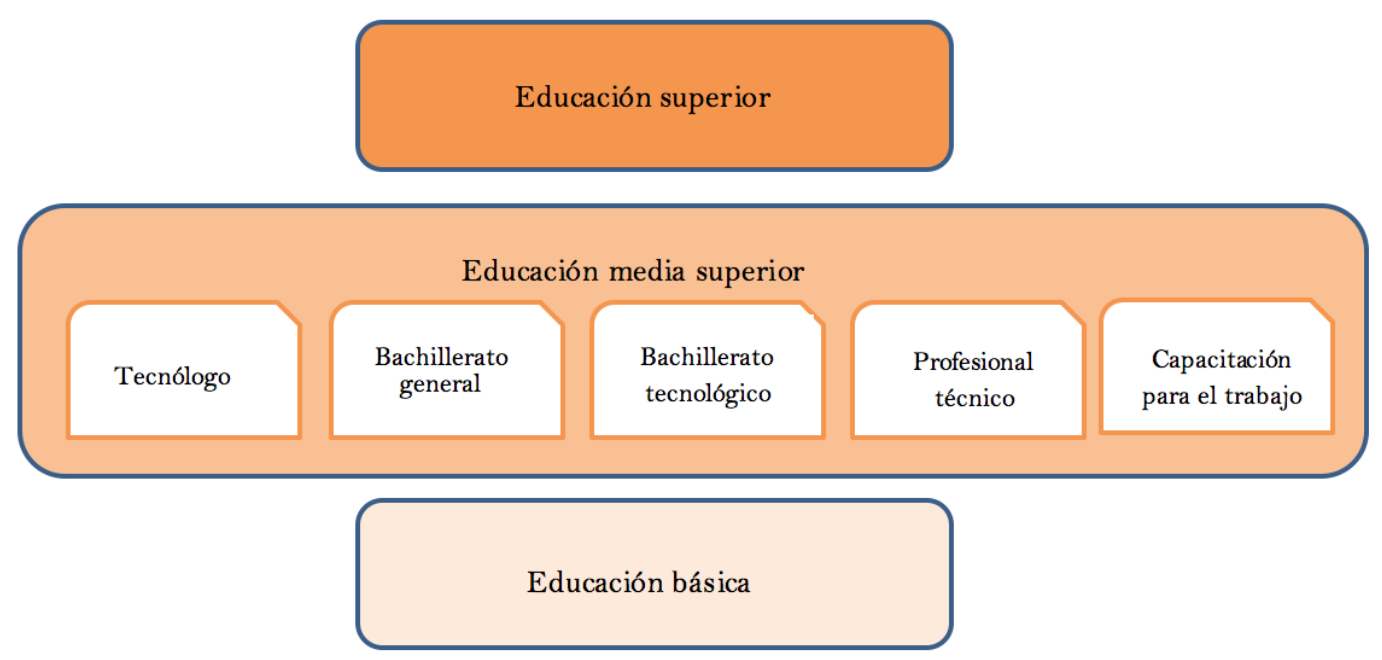

Figura 1. Ubicación y estructura de la educación media superior dentro del Sistema Educativo Nacional

Fuente: Elaboración propia con base en SEMS (2013b).

Los distintos subsistemas varían en cuanto a sus objetivos, organización escolar, currículo y el tipo de formación de los alumnos. A los tres ya existentes, el bachillerato general, el bachillerato tecnológico y el profesional técnico, la reforma de 2013 incluyó el tecnólogo y la capacitación para el trabajo. El bachillerato general incluye las modalidades de preparatoria abierta y a distancia. El bachillerato tecnológico ofrece la carrera de técnico profesional y prepara para la continuación de estudios del tipo superior. La educación profesional técnica forma profesionales calificados en diversas especialidades (DGAIR, 2000).

En el aspecto administrativo, se requiere contar con el certificado de secundaria para ingresar a la educación media superior. La mayoría de las escuelas -privadas y públicasrequieren presentar un examen de admisión. Por lo general, se sigue un plan de estudios de tres años, pero también existe el de dos. El objetivo principal del bachillerato general es preparar a los estudiantes para continuar estudios superiores. Esta modalidad es de carácter formativo e integral, comprende conocimientos científicos, técnicos y humanísticos, así como algunas metodologías de investigación y de dominio del lenguaje. Además,

promueve que el estudiante asimile y participe en los cambios que acontecen en su entorno, en su país y en el mundo. También se busca dotar al bachiller de la capacidad para manejar algunas herramientas adecuadas para el análisis y la resolución de problemas, así como ofrecerle una formación que corresponda a las necesidades de su edad [...] Los formatos de certificación para el bachillerato general escolarizado son [el] certificado de terminación de estudios [y la] certificación de estudios. (DGAIR, 2000, p. 12) 


\section{El derecho a la educación y su correlación con las obligaciones del gobierno ante los datos de la educación media superior}

Al confrontar los datos que arroja el estado de la educación media superior en México con las dimensiones del derecho a la educación a la luz del esquema de las "4 As" de Tomasevski (2004), se identifica la violación de este derecho, y la complejidad que enfrenta este nivel educativo.

\subsection{Asequibilidad}

De acuerdo con Tomasevski (2004), en esta dimensión el gobierno tiene la obligación de asegurar la educación gratuita y obligatoria a todos los jóvenes en edad escolar y hasta, por lo menos, la edad mínima para emplearse; así como la de respetar la libertad de los padres para escoger la educación de sus hijos.

Las recientes reformas han representado avances en cuanto a la formulación jurídica del derecho a la educación, la gratuidad y la obligatoriedad de la misma. Sin embargo, la numeraria nos permite ver vacíos importantes en el cumplimiento de este derecho. Hasta el ciclo escolar 2012-2013, la matrícula en educación media superior correspondía a 12,6\% de la matrícula total nacional (desde preescolar hasta nivel superior, desagregando capacitación para el trabajo). La población se encontraba dividida en $49 \%$ de hombres y $51 \%$ de mujeres (figura 2 )

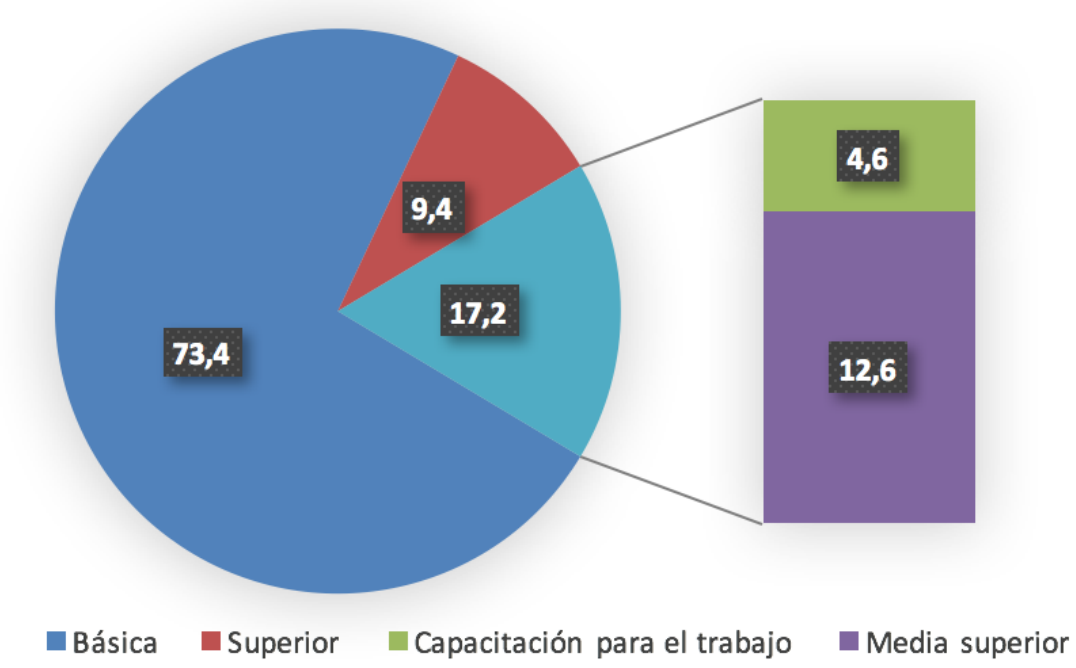

Figura 2. Matrícula total nacional por nivel educativo en porcentajes Fuente: Elaboración propia a partir de SEMS (2013c).

En alineación con las políticas educativas implementadas para este nivel, uno de los aspectos que llama la atención en el marco del derecho a la educación es la cobertura. El Sistema Nacional de Indicadores Educativos (SNIE, 2013) pondera una cobertura en el ciclo actual (2016-2017) de 77,8\%. Se estima así que se encuentran en el sistema educativo a nivel medio superior casi 8 de cada 10 jóvenes en edad de cursarlo. Sin embargo, datos de la OCDE señalan que la tasa de matriculación en México entre jóvenes de 15 a 19 años es la tercera más baja entre los países miembro, 55,6\%, ubicada en el lugar 39 de 41 (OCDE, 2016a). Dicha cobertura no ha equivalido a gratuidad en todos los casos. De la matrícula 
total nacional, $83 \%$ pertenece al sector público y $17 \%$ al privado (figura 3.) La atención en cada uno de los tipos de sostenimiento tampoco es equitativa. Los datos más recientes de la SEP reportan tasas de una escuela por cada 347 educandos, un grupo por cada 36 estudiantes y un docente por cada 19 alumnos en régimen público, y una escuela por cada 141 educandos, un grupo por cada 21 estudiantes y un docente por cada 8 alumnos en sostenimiento privado (figura 4).

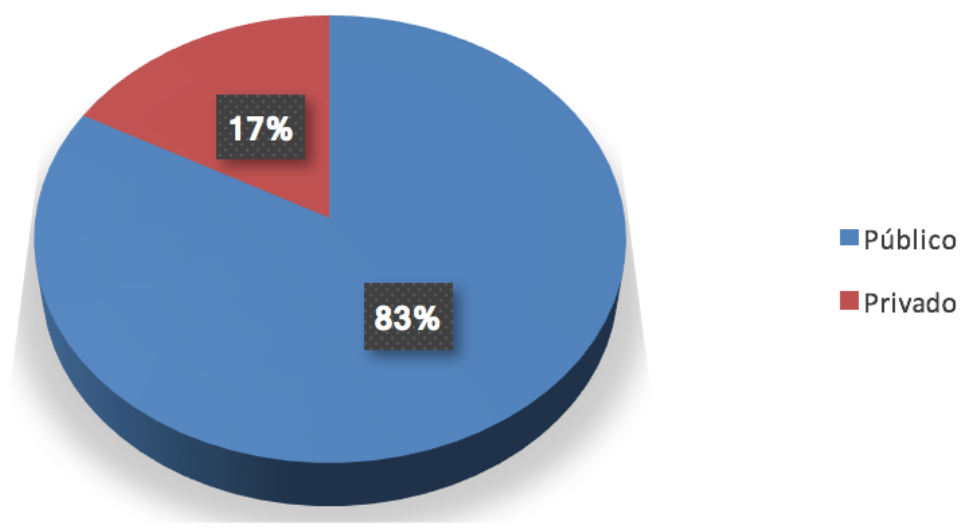

Figura 3. Matrícula total nacional por sector Fuente: Elaboración propia a partir de SEMS (2013c).

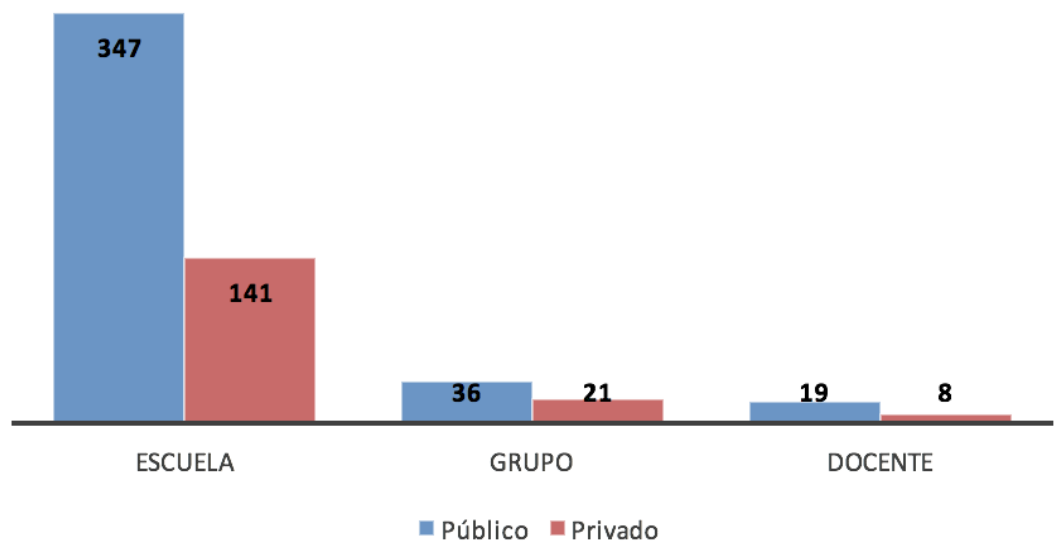

Figura 4. Tasa de alumnos por escuela, grupo y docente según tipo de sostenimiento Fuente: Elaboración propia a partir de SEMS (2013c).

Por otro lado, parte del 17\% de cobertura de que da cuenta el sector privado no representó de inicio la primera opción de los alumnos y sus padres, sino que fue resultado del rechazo de aquéllos en su centro de elección. El desembolso en colegiaturas en estos casos representa un gasto excedente al presupuesto familiar que en ocasiones tendrá que subsanar el propio estudiante dedicando parte de su tiempo a trabajar y cayendo así en mayor riesgo de reprobación, rezago o deserción definitiva. El reporte de la COPEEMS (2014) expone que la cantidad de jóvenes que reportó falta de dinero para pasajes, colegiaturas o inscripción es casi 50 puntos porcentuales mayor en el caso de los provenientes de hogares con ingresos más bajos en relación con los de ingresos más altos.

Si bien la cobertura se relaciona con una distribución equitativa del bien social que significa la educación y, en términos de Pablo Latapí (1993), esto corresponde a cierto 
grado de justicia educativa, ésta de hecho no equivale ni garantiza el acceso a la educación u otras dimensiones del derecho a la educación, como la calidad, la igualdad de oportunidades, la equidad, el derecho de aprender, entre otras. De estos aspectos pueden dar cuenta otros indicadores educativos como el rezago, los porcentajes de reprobación y de deserción y la tasa de terminación. Las razones que determinan estos indicadores son variadas y atañen de manera transversal a diversas dimensiones del derecho a la educación.

Asimismo, la gratuidad vinculada al derecho a la educación tiene como fundamento el ofrecer igualdad de oportunidades, en especial en el acceso a la misma. Sin embargo, sus efectos para el ejercicio de ese derecho quedan en entredicho cuando se identifica que quienes presentan rezago educativo por extraedad grave y se encuentran en localidades rurales de alta marginación se ubican en su mayoría dentro del sector privado (cuadro 1)

Cuadro 1. Porcentaje de alumnos con extraedad grave en educación media superior por sostenimiento, tamaño de localidad y grado de marginación (2013-2014)

\begin{tabular}{lccccccc}
\hline & \multicolumn{2}{c}{ URBANO } & \multicolumn{2}{c}{ RURAL } & \multicolumn{2}{c}{ No } & \multirow{2}{*}{ TotaL } \\
\cline { 2 - 5 } & $\begin{array}{c}\text { Baja } \\
\text { margin. }\end{array}$ & $\begin{array}{c}\text { Alta } \\
\text { margin. }\end{array}$ & $\begin{array}{c}\text { Baja } \\
\text { margin. }\end{array}$ & $\begin{array}{c}\text { Alta } \\
\text { margin. }\end{array}$ & CLASIFICADo & \\
\hline Público & 13,3 & 11,7 & 12,6 & 13,9 & & 12,0 & 13,0 \\
Privado & 26,6 & 22,2 & 13,6 & 16,8 & & 14,5 & 26,0 \\
Total & 16,7 & 12,4 & 12,7 & 14,0 & & 12,3 & 15,5 \\
\hline
\end{tabular}

Nota: Los niveles de marginación medio, bajo y muy bajo se encuentran agrupados en baja marginación; los niveles alto y muy alto se encuentran agrupados en alta marginación.

Fuente: Síntesis de la tabla Porcentaje de alumnos con extraedad grave en educación media superior por sostenimiento, control administrativo, tamaño de localidad y grado de marginación en INEE (2015).

A detalle, los datos del cuadro 1 muestran que 15,5\% de la población en media superior se encuentra en extraedad grave, con una mayor incidencia en centros educativos privados en localidades urbanas de baja marginación lo cual siembra la inquietud de saber tanto las causas del rezago de esos alumnos como las de la absorción de los mismos en el régimen privado. En zonas rurales de alta marginación se puede observar asimismo que la mayor incidencia de extraedad grave recae de nuevo en el sector privado, planteando las mismas dudas. En el reporte del INEE (2015) de donde se obtienen dichos datos, se muestra asimismo que $23 \%$ de los alumnos en edad de cursar el nivel medio superior se encontraban en rezago ligero (un grado escolar inferior al que deberían estar cursando según su edad idónea o típica), mientras que $7.9 \%$ se encontraban en rezago grave y aún seguían cursando algún grado de secundaria.

La obligatoriedad presenta una circunstancia similar. Si bien la tasa de absorción que se expresa en el Reporte de la Encuesta Nacional de Deserción en la Educación Media Superior (COPEEMS, 2014) es de 99.5\%, cabe señalar que las cifras consideran a los rezagados de otros ciclos de secundaria, por lo que hay que tomarlas con cautela y sin falso optimismo. Las altas tasas de absorción y cobertura ocultan, respectivamente, un proceso de rezago grave y una tasa neta de cobertura por parte del sector público de $65 \%$ (figura $3)$.

Del mismo modo, la eficacia y equidad en la cobertura tendría que responder en parte por el $14.9 \%$ de alumnos que deserta y a quienes no se les aseguraron las condiciones necesarias para su permanencia. De la mano de este punto va la exigibilidad del derecho a la educación, en ambas vías, tanto para el Estado como garante de los derechos de sus 
ciudadanos, como para los alumnos como ejecutores de los mismos. Si bien la deserción en educación media superior puede llegar a ser una decisión tomada en libertad, el arrepentimiento posterior y el enfrentarse a un limitado campo de trabajo como consecuencia de la misma llama a cuestionar cuáles son las herramientas y las estrategias para dar viabilidad y cause a la exigibilidad de ese derecho. ¿A quién se le exige la mejora de resultados? ¿Cómo se exige que se hagan patentes? Ante qué autoridad se pone la queja de no estar recibiendo la educación que se requiere, a pesar incluso de haber migrado al sector privado y haber renunciado ya al derecho a la gratuidad en la educación. El presupuesto en inversión social asignado a educación no contempla una partida para defensoría de los derechos de los alumnos. No existe un órgano rector al respecto. $\mathrm{Al}$ parecer se ha dejado la tarea para los órganos defensores de los derechos humanos, de los derechos de las niñas, niños y adolescentes o, en ocasiones, defensores de los pueblos.

Datos de la OCDE señalan que el porcentaje de jóvenes que se espera que se gradúen del nivel medio superior es uno de los más bajos, 51,3\%, ubicado en el lugar 35 de 37 naciones (OCDE, 2016a). Asimismo, informa que, aunque la población de 25 a 34 años en México que alcanzó la educación media superior ha aumentado en los últimos años, de $38 \%$ a $46 \%$, "este porcentaje es mucho menor que el promedio de la OCDE de $83 \%$. Sólo uno de tres adultos de 25 a 64 años de edad terminó ese nivel de educación” (OCDE, 2016b, p. 1). Este último dato es muy cercano al de eficiencia terminal reportado por el SNIE, que es de $67,2 \%$ (SNIE, 2013).

A estos datos, se agregan los referentes a la deserción escolar. El Reporte de la Encuesta Nacional de Deserción en la Educación Media Superior (COPEEMS, 2014) muestra que sólo 36 de cada 100 alumnos culminan su trayectoria escolar de manera satisfactoria. El mismo reporte de la COPEEMS expresa una tasa de deserción en nivel medio superior de 14,9\% y da cuenta de las principales causas de ella. A saber, la situación económica es la razón dominante manifiesta para abandonar la escuela, independientemente de sector social, edad o género. Le siguen el embarazo, tener un hijo o casarse, la reprobación de materias y el disgusto por el estudio. Quienes desertaron contaban con menor promedio que quienes no lo hicieron. El 69,7\% de los jóvenes que desertaron considera que fue una mala o muy mala decisión dejar de ir a la escuela. El 42,8\% percibe que la confianza en sí mismo fue afectada negativamente por haber dejado de estudiar. El $60,5 \%$ piensa que la posibilidad de encontrar trabajo fue afectada negativamente por haber dejado de estudiar. Los incentivos más mencionados por los desertores para continuar con sus estudios fueron recibir un apoyo económico, tener un programa de estudios interesante y contar con opciones educativas con horarios y programas flexibles.

Sin embargo, retomando a Latapí (1993) ha de recordarse que el bien social denominado educación se distingue de otros bienes no sólo por su inmaterialidad, sino por la asimilación del mismo en el propio sujeto y, lo más importante, por parte del propio sujeto: "la educación supone siempre un esfuerzo personal; no es un bien que se dé; es una tarea que realiza cada persona, con ciertos apoyos y recursos que no determinan los resultados" (p. 25). En congruencia a ello, se trata de un derecho que, como tal, no se recibe, sino que se ejerce. El papel activo del alumno es central. Debe considerarse y capitalizarse el perfil psicológico del adolescente. En la misma línea, Amézquita (2014) destaca que

la valoración del derecho a la educación en los sujetos debe darse desde las vivencias situadas y diferenciadas de los y las estudiantes en el contexto escolar. [...] Es en el contexto escolar donde las y los estudiantes construyen sus propias comprensiones y 
expectativas respecto a la educación [...] En tanto sujetos del derecho a la educación sus voces requieren ser escuchadas. (pp. 101, 104)

y, más que eso, atendidas. El alumno de nivel medio superior ha recorrido ya por lo menos la media de escolaridad del resto de los mexicanos, es decir, nueve años. Su experiencia le ha conformado una actitud de vida y una actitud ante el estudio y el entorno escolar. ¿Cómo se ha construido su experiencia hasta ese momento? El reporte de la COPEEMS (2014) grafica un dato abrumador: 30,1\% de los desertores entrevistados y 17,9\% de los no desertores entrevistados declararon tener problemas para entenderle a los maestros, lo cual de manera agregada nos da un aproximado a $20 \%$ del total de la matrícula con esa incidencia, además de permear que en términos numéricos son muchos más los no desertores que así lo afirmaron.

\title{
2.2. Accesibilidad y aceptabilidad
}

En opinión de Tomasevski (2004), en la dimensión de accesibilidad el gobierno tiene la obligación de eliminar la exclusión de la escuela por razones internacionalmente prohibidas (raza, color, sexo, lengua, religión, opinión política, posición económica, entre otras); así como la de eliminar la discriminación racial y de género, asegurando en la práctica el igual disfrute de los derechos humanos. Por su parte, la aceptabilidad requiere del gobierno la obligación de establecer estándares mínimos para la educación, incluyendo los medios de instrucción, contenidos y métodos de enseñanza, asegurando que todas las instituciones cumplan con esos estándares; así como la de mejorar la calidad de la educación, asegurando que el sistema educativo sea acorde con los derechos humanos. Ambos aspectos se consideran en este apartado dado que los mismos indicadores educativos dan cuenta de uno y otro, así como por estar integrados en la política educativa actual.

La política educativa mexicana colocó en la agenda pública los elementos básicos del derecho a la educación: la equidad, la calidad, la igualdad de oportunidades, la universalidad y el derecho a aprender. Si se parte de que la equidad educativa supone que se le puede considerar un concepto que engloba al resto porque

\begin{abstract}
determinados bienes educativos sean distribuidos de forma equitativa, por ejemplo, los recursos, la calidad del profesorado, la oferta educativa, cuidando que las desigualdades no condicionen el aprendizaje y el rendimiento escolar, y poniendo los medios y recursos compensatorios en los alumnos desfavorecidos natural o socialmente. (Bolívar, 2005, p.4)
\end{abstract}

De cualquier manera, es preciso puntuar las deficiencias en cada uno de ellos. Asimismo, identificar que, si bien entre estas dimensiones a la que se le ha brindado más atención ha sido a la calidad educativa, la que representa mayores retos es la igualdad de oportunidades. Solís (2010) destaca que, si bien las
brechas educativas se originan en las diferencias interpersonales, (...) también se asocian a las dificultades propias de la condición social y a las deficiencias institucionales, que transforman las desventajas de origenes sociales en falta de oportunidades educativas. En este sentido, resolver las desigualdades educativas persistentes implica en gran medida reconocer, dimensionar y resolver el problema de la desigualdad social. (pp. 600-601)

Este aspecto es una constante que atraviesa todos los indicadores educativos. En lo que se refiere a absorción y cobertura, las poblaciones indígenas y con alguna discapacidad que no acceden al nivel medio superior casi duplican a las de la población en general. La población que cae en rezago y deserción en su mayoría pertenece a sectores vulnerables 
por condición socioeconómica o étnica. Tan sólo en la Ciudad de México, por ejemplo, Yanes y Peralta (2006) refieren que a 2005, del 10\% de la población entre 15 y 19 años hablante de alguna lengua indígena, $72,4 \%$ no asistía a la escuela, entre los cuales se encontraban 8 de cada 10 mujeres. Asimismo, del 6,9\% de la población con alguna discapacidad en esa entidad, más de la mitad no asistía a la escuela. Para estos jóvenes, los contenidos y la manera en que se presentan, carecen de pertinencia. Se ha intentado atacar este punto con políticas educativas compensatorias, no obstante, habrá que revisar la eficacia real de las mismas. Muñoz y Núñez (2004) advierten que, aunque programas como el Progresa fueron exitosos

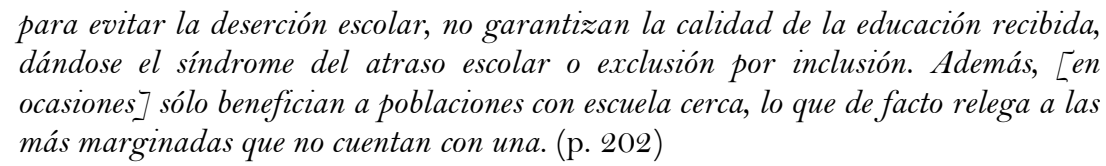

Los alcances de los programas llevados a cabo, concluyen, siguen dejando mucho que desear en cuanto a derecho a la educación, justicia y equidad se refiere.

En términos numéricos, los indicadores educativos en nivel medio superior permiten identificar el incumplimiento en las dimensiones de accesibilidad y aceptabilidad del derecho a la educación en el nivel medio superior. En la figura 5 se observa que en materia de cobertura se enfrenta el enorme reto de mejorar la eficiencia terminal y evitar el importante rezago que existe al integrar los indicadores de rezago ligero y extraedad grave.

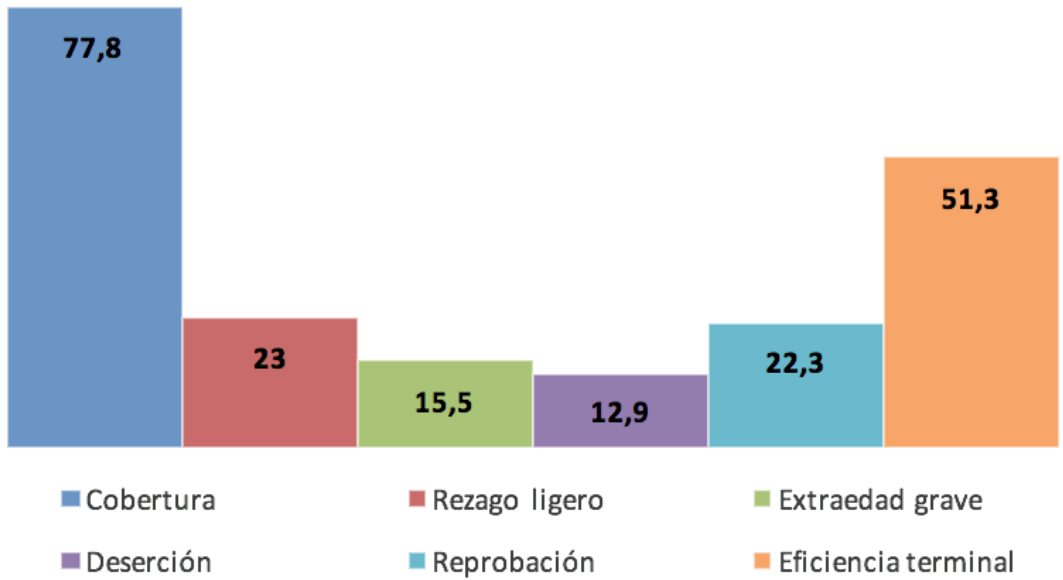

Figura 5. Indicadores educativos en educación media superior, en porcentajes a 20132015

Fuente: Elaboración propia con datos de SNIE (2013), INEE (2015), COPEEMS (2014) y OCDE (2016a).

Los datos sobre deserción, las causas de ella y sus efectos en el ámbito personal y laboral dan cuenta de la problemática educativa de este nivel, los cuales deben considerarse como asuntos de primordial relevancia para las políticas públicas en general y no sólo la educativa, recordando que "las desigualdades educativas se originan en reductos del tejido social, -en ocasiones- inaccesibles a la acción del poder central” (Latapí, 1993, p. 29). Por otro lado, la tasa de aprobación nacional para el ciclo 2012-2013 en nivel medio superior fue de $85,3 \%$ (INEE, 2015) lo cual indicaría que 15 de cada 100 alumnos no aprueban el 
grado escolar que cursan. Empero, los pronósticos del SNIE para el ciclo escolar 20162017 expresan un porcentaje de reprobación mucho mayor, de 29,9\% (SNIE, 2013)

Por su parte, el índice de reprobación remite ineludiblemente a los resultados del Informe PISA 2015, en los que 33,8\% de los evaluados obtuvo un rendimiento bajo (OCDE, 2016c). Esto equivaldría a pensar que, de los 36 alumnos en promedio en un grupo de preparatoria pública, 12 no realizan operaciones matemáticas básicas, no leen de manera eficiente ni cuentan con conocimientos básicos de ciencias. Los resultados de la prueba PLANEA (SPEC, 2016) son incluso más desfavorecedores. En el nivel más bajo en matemáticas se encuentra $49,2 \%$ de los jóvenes, y en el más bajo de lenguaje y comunicación, $45 \%$ de ellos. Chiapas se caracteriza por ser el estado con mayor porcentaje de estudiantes en el nivel más bajo $(65,2 \%)$ y el menor porcentaje en el nivel más alto $(3,5 \%)$. Ante estos hechos, se abren importantes cuestionamientos en torno a los beneficios de la cobertura resultante de la obligatoriedad del nivel medio superior. Zorrilla (2005) apunta a la importancia de darle un sentido formativo al bachillerato, un sentido propio y no uno como de paso. El sistema educativo, de acuerdo al mismo autor, tal como está divide a la sociedad y no queda una clase media educativa, sólo la élite que logra egresar del nivel medio superior y los que son menospreciados por considerar que no sirven para nada. Aboites (2012) por su parte, observa que

la renuencia del Estado a financiar el crecimiento de la educación pública y [definir] las limitaciones de la privada para ofrecer un lugar y una buena instrucción a la mayoría se combinan para generar un claro deterioro en la posibilidad de ejercer el derecho a la educación. (p. 374)

En conjunto, estas dimensiones hablan de una brecha educativa importante.

Sin bien datos de la OCDE indican que el gasto público en educación en México es el segundo mayor entre los países miembro, con 17,3\% del gasto público total, (OCDE, 2016a) a su vez el gasto público nacional es uno de los más bajos en relación al producto interno bruto (PIB) entre los países considerados. Por su parte, el Centro de Estudios Sociales y de Opinión Pública (Cesop, 2015) informa que, del presupuesto para educación en 2016, el $11,8 \%$ corresponde al nivel medio superior. Del mismo, 67,2\% se destinó a los alumnos; $31,7 \%$, a la infraestructura física; 0,6\%, a los docentes, y 0,5\% a la gestión escolar. Estas cifras abren importantes cuestionamientos ya que no se desagregan, por un lado y, por el otro, existen rubros vitales como la capacitación docente que no se sabe si queda fuera, como parte de alguno de los otros mencionados, o como responsabilidad y erogación propia de cada uno de los profesores. La suma designada a los alumnos tampoco da cuenta de cómo se distribuye, si considera el monto en becas, si es el monto calculado del gasto por alumno o de qué forma es que una proporción tan alta llega a ellos, pues se está hablando de alrededor de 60 millones de pesos repartidos entre poco menos de cinco millones de alumnos. Ibagón (2015), entre otros, ha señalado la importancia de la distribución presupuestaria de los recursos públicos destinados a educación, ya que la misma tiene un impacto directo en la reducción o ampliación de las brechas educativas al interior de una sociedad.

Por otro lado, la inversión en tiempo que pasan los maestros dando clase a grupo es la tercera más intensa de los países de la OCDE, con un total de 848 horas (OCDE, 2016a). Al igual que en el caso de los recursos económicos, la cantidad de ninguna manera ha equivalido a calidad. Algunos de los aspectos que explican esto fueron revelados en el reporte de la Cesop (2015), los cuales se enumeran a continuación: 
- En el 2012 no se habían homologado los contenidos curriculares de los planes y programas de estudio en todas las modalidades y subsistemas que integran la Educación Media Superior.

- De 2008 a 2012, el 57,6\% de los docentes de Instituciones Públicas de Educación Media Superior se inscribió al Programa de Formación Docente de Educación Media Superior; el 36,5\% egresó, y sólo el 8,6\% certificó las competencias adquiridas, establecidas en el perfil docente de Educación Media Superior.

- A 2014, la SEP no contó con un censo sobre el perfil y nivel académico docente, ni con un diagnóstico de las necesidades de actualización, lo que limitó la operación de servicios para mejorar su desempeño.

- Los resultados del concurso de ingreso al Servicio Profesional Docente evidenciaron que, de los 34.639 sustentantes en 2014 , el $67,2 \%$ fue calificado como no idóneo.

- A 2014, la SEP no dispuso de un diagnóstico integral de las condiciones físicas y de equipamiento de los 17.245 planteles públicos de Educación Media Superior.

De ninguna manera se puede atribuir exclusivamente al desempeño docente la responsabilidad en la calidad de la educación media superior, pero al comparar este listado con las causas de deserción escolar es imposible soslayar su relación con dos de las principales: la reprobación de materias y el disgusto por el estudio. El reporte de la COPEEMS (2014) desarticula en una matriz los distintos factores exógenos y endógenos para la deserción (cuadro 2) y en ella se detectan coincidencias con el diagnóstico de la Cesop. Entre ellas, sobresalen las relativas a la propuesta curricular y metodológica, las relativas a equipamiento e infraestructura escolar, así como a la planta docente y el capital cultural de los mismos. El trabajo de la COPEEMS (2014) concluye que

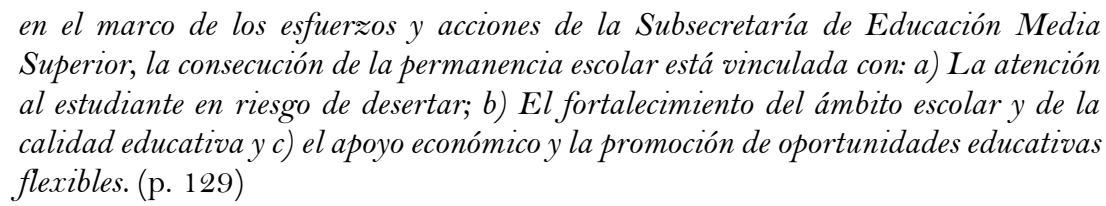

Con todo, se ha recurrido a la evaluación como un medio para asegurar la calidad educativa. De hecho, tanto la evaluación a alumnos como a docentes develan un panorama del incumplimiento del derecho a aprender. La evaluación misma ha sido sujeta a fuertes cuestionamientos sobre su pertinencia y, sobre todo, su equidad. Chizzotti (2016), por ejemplo, advierte que los procesos de evaluación corrientes y los conceptos erróneos discriminatorios de evaluaciones meramente acumulativas, tienden a clasificar a los estudiantes. Él evalúa el sesgo punitivo de notaciones meritocráticas de aprendizaje y argumenta que sólo la evaluación formativa puede garantizar el derecho a aprender. Asimismo, destaca que

La Conferencia Internacional sobre el aprendizaje en el siglo XXI indica seis principios fundamentales para la evaluación formativa de los estudios de investigación y de casos: el establecimiento de una cultura de clase que fomente la interacción y el uso de herramientas de evaluación; la definición de los objetivos de aprendizaje y el seguimiento del progreso individual de los estudiantes hacia estos objetivos; el uso de métodos de enseñanza variados para satisfacer las diversas necesidades de los estudiantes; recurrir a métodos también diversos para evaluar los resultados de los estudiantes; dar retroalimentación del desempeño de los estudiantes 
y adaptar la enseñanza para satisfacer las necesidades identificadas, y, por último, la participación activa del estudiante en el proceso de aprendizaje. (p. 567)

Cuadro 2. Matriz de análisis de factores asociados al fracaso escolar

\begin{tabular}{|c|c|c|}
\hline DIMENSIÓN & FACTORES EXÓGENOS & FACTORES ENDÓGENOS \\
\hline $\begin{array}{l}\text { Material/ } \\
\text { Estructural }\end{array}$ & $\begin{array}{l}\text { Nivel socioeconómico de la familia. } \\
\text { Escolaridad de los padres y de adultos del } \\
\text { hogar } \\
\text { Composición familiar } \\
\text { Características de la vivienda } \\
\text { Grado de vulnerabilidad social (desempleo, } \\
\text { consumo de drogas, delincuencia, etc.) } \\
\text { Origen étnico } \\
\text { Situación nutricional de los niños } \\
\text { Trabajo infantil y de los adolescentes }\end{array}$ & $\begin{array}{l}\text { Equipamiento - infraestructura } \\
\text { escolar } \\
\text { Planta docente } \\
\text { Material educativo } \\
\text { Programas de alimentación y } \\
\text { salud escolar } \\
\text { Becas }\end{array}$ \\
\hline $\begin{array}{l}\text { Política/ } \\
\text { Organizativa }\end{array}$ & $\begin{array}{l}\text { La estructura del gasto público } \\
\text { Conjunto de políticas económicas o } \\
\text { sociales que inciden en las condiciones en } \\
\text { que los niños llegan a la escuela } \\
\text { Tipo de organizaciones y redes } \\
\text { comunitarias incentivadas, a través de } \\
\text { lineamientos y programas públicos y/o } \\
\text { de la sociedad civil } \\
\text { Políticas dirigidas al mejoramiento de } \\
\text { condiciones económicas y laborales de las } \\
\text { minorías étnicas y grupos vulnerables } \\
\text { Estrategias no gubernamentales } \\
\text { orientadas a promover la escolarización y } \\
\text { permanencia en el sistema }\end{array}$ & $\begin{array}{l}\text { Grado de descentralización del } \\
\text { sistema escolar } \\
\text { Modalidad de financiamiento } \\
\text { para la educación } \\
\text { Estructura del sistema educativo } \\
\text { Articulación entre los diferentes } \\
\text { niveles de gobierno } \\
\text { Propuesta curricular y } \\
\text { metodológica } \\
\text { Mecanismos de supervisión y } \\
\text { apoyo a los establecimientos } \\
\text { Situación de los docentes en } \\
\text { cuanto a formación, } \\
\text { actualización y condiciones } \\
\text { laborales } \\
\text { Articulación con otros actores } \\
\text { extraeducativos }\end{array}$ \\
\hline Cultural & $\begin{array}{l}\text { Actitud, valoración hacia la educación } \\
\text { Pautas de crianza y socialización } \\
\text { Consumos culturales } \\
\text { Pautas lingüísticas y de comunicación al } \\
\text { núcleo familiar } \\
\text { Expectativas y aspiraciones } \\
\text { Capital cultural de las familias } \\
\text { Uso del tiempo de los niños y de los } \\
\text { jóvenes }\end{array}$ & $\begin{array}{l}\text { Capital cultural de los docentes } \\
\text { Estilo y prácticas pedagógicas } \\
\text { Valoración de expectativas de los } \\
\text { docentes y directivos respecto } \\
\text { de los alumnos } \\
\text { Clima y ambiente escolar } \\
\text { Liderazgo y conducción }\end{array}$ \\
\hline
\end{tabular}

Fuente: Elaborado a partir de COPEEMS (2014).

Es innegable que la educación, en especial la media superior, se encuentra inserta en un proceso de evaluación acumulativa neto. Los alumnos lo enfrentan desde el examen de ingreso (EXANI-I) gestionado por una instancia privada como lo es el Ceneval, las evaluaciones nacionales e internacionales (PLANEA y PISA, respectivamente), la seriación de materias que determina la aprobación de grado y en su conjunto, la obtención del certificado. Por su parte, los docentes en este nivel ya están jurídicamente vinculados a la evaluación como los de nivel básico, mediante el Artículo 52 de la Ley General del Servicio Profesional Docente, el cual señala que la evaluación del desempeño es obligatoria para los docentes y técnicos docentes en servicio de Educación Media Superior, y que se realizará por lo menos cada cuatro años.

Los productos de las pruebas estandarizadas reflejan una importante brecha en los resultados educativos que nace de la desigualdad de oportunidades. En el caso de PLANEA, por ejemplo, al hacer una deducción simple de los alumnos en nivel satisfactorio frente a 
los del nivel más bajo, se da una diferencia de $-39,5$ puntos en escala nacional. Para el caso de Chiapas, sin embargo, la misma deducción da un resultado de $-58,2$ puntos (figura 6 ) En cuanto al tipo de subsistema, el bachillerato intercultural presenta una diferencia de 73,6 puntos. La diferencia en sector privado sigue siendo negativa, pero menor que la nacional, de -33,8 puntos. Cabe mencionar que el único subsistema en el país con diferencia positiva, y muy pronunciada, es el CETI, con 21,3 puntos.

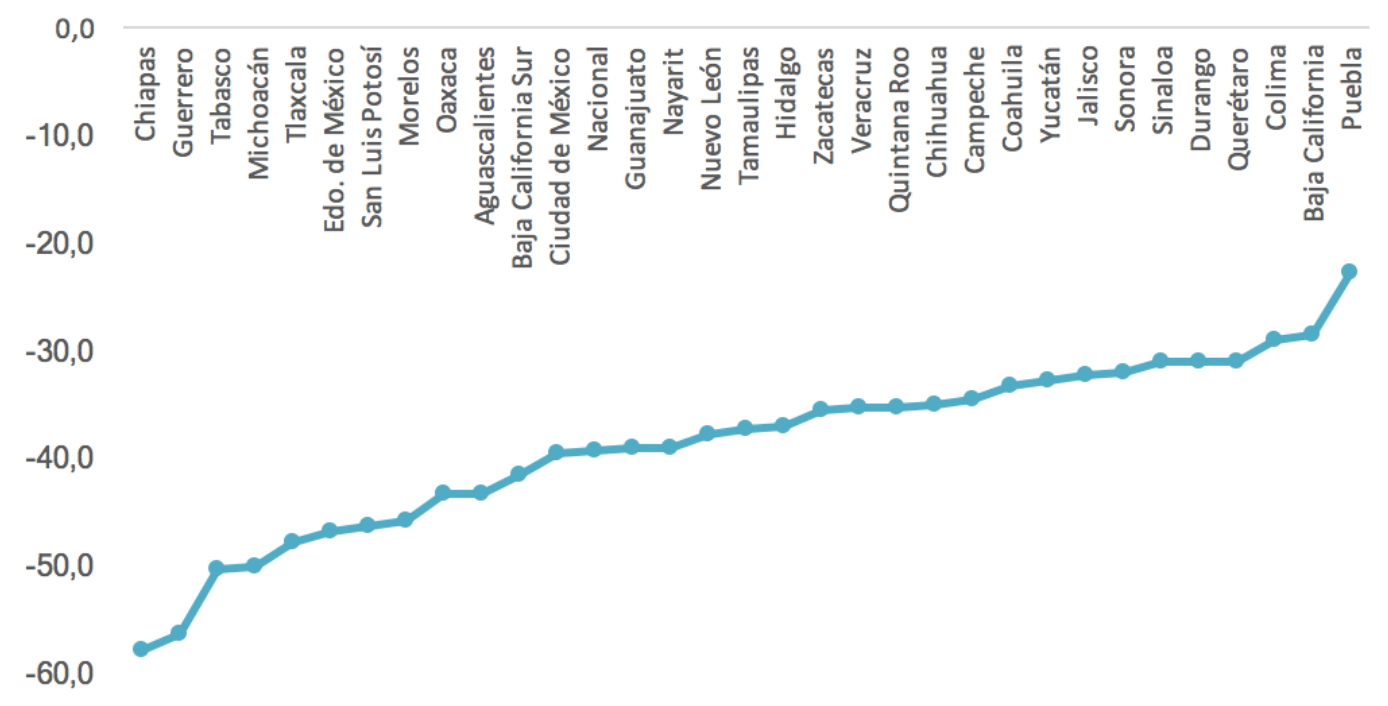

Figura 6. Brecha de resultados entre alumnos con nivel de logro mayor y menor en la prueba PLANEA 2015 por entidad federativa

Fuente: Elaboración propia con datos de SPEC (2016).

\subsection{Adaptabilidad}

Para Tomasevski (2004), en esta dimensión el gobierno tiene la obligación de diseñar e implementar educación para atender a los jóvenes excluidos de la educación formal (migrantes, trabajadores, etc.); la de adaptar la educación para los jóvenes con discapacidad, las minorías y los indígenas; así como la de garantizar todos los derechos humanos dentro de la educación y mejorar los derechos humanos a través de la educación.

Este aspecto está íntimamente relacionado con el derecho humano a aprender. El enfoque por competencias del MCC tuvo como objetivo fomentar aprendizajes pertinentes que cobren significado en la vida real de los estudiantes por medio de competencias genéricas clave, transversales y transferibles. Las competencias definidas como clave son aquellas aplicables en contextos personales, sociales, académicos y laborales amplios; así como las relevantes a lo largo de la vida. Las competencias se consideran transversales cuando son relevantes a todas las disciplinas académicas, así como a las actividades complementarias y procesos escolares de apoyo a los estudiantes. Por su parte, son transferibles las que refuerzan la capacidad de adquirir otras (DOF, 2008) No obstante, al contrastarlas con las causas de la deserción y las tasas de reprobación ya revisadas, se puede observar que sus alcances reales han sido limitados.

La universalidad, explica Tomasevski (2004), es un concepto que parte de la legislación internacional y que se basa en la cooperación internacional, con el objetivo de alcanzar la igualdad de oportunidades para disfrutar el derecho a la educación. Para cumplir dicho fin, se asignan recursos adicionales a los países, comunidades y familias más pobres. Sin 
embargo, la autora deja claro que para que este concepto se logre es indispensable que las correspondientes obligaciones gubernamentales, individuales y colectivas también sean universales. En este sentido de corresponsabilidad, es de llamar la atención la experiencia del colectivo Movimiento de Aspirantes Excluidos de la Educación Superior (MAES, 2015), el cual identifica como su objetivo fundamental

\begin{abstract}
la transformación de la política educativa vigente -en lucha-por otro país y por un futuro que nos incluya a todos los jóvenes, por la defensa de la educación pública y por el derecho a recibir educación media superior y superior gratuita y de alto nivel académico y que desde 2006 han logrado avances importantes, como conseguir que cientos de jóvenes ingresen a la UNAM, al Politécnico, la UPN, la UAEM y la UACM por otra vía que no es la del examen de admisión. (p. 1)
\end{abstract}

Un primer paso para afirmar la universalidad del derecho a la educación, así, sería vetar las prácticas discriminatorias. Por desgracia, los distintos indicadores educativos (figura 5) revisados dejan claro que, desde nivel escolar hasta el gubernamental e internacional, no se ha alcanzado este ideal.

\title{
3. A manera de conclusiones
}

El derecho a la educación en nivel medio superior representa un ámbito políticopedagógico para su exigibilidad y justiciabilidad. La sociedad desempeña un papel importante para visibilizar las carencias que existen todavía en este ámbito, de manera que se incluyan y se mantengan en la agenda pública y así exigir de forma activa el aseguramiento del derecho a la educación. Por otra parte, toca a las autoridades establecer los mecanismos necesarios para que se haga justicia cuando se caiga en omisión de dichos derechos. El modelo de las " 4 As" permite visibilizar la situación del derecho a la educación en nivel medio superior en México. La atención generalizada en el nivel básico ha soslayado los esfuerzos hacia la educación media superior. El nivel medio superior estuvo inserto en procesos de reforma desde 1992 y su última modificación fue en 2013. De estos cambios los más relevantes fueron los relativos al modelo por competencias, al marco curricular común y el que hizo obligatoria la educación media superior. La estructura de los distintos subsistemas no tuvo grandes cambios, sólo se anexaron las modalidades de tecnólogo y la capacitación para el trabajo. Tampoco hubo cambios importantes en cuanto a los requisitos de ingreso y egreso.

A diez años de la RIEMS, sus cuatro pilares -la construcción de un marco curricular común, la definición y reconocimiento de las opciones de oferta, la profesionalización de los servicios educativos y la certificación nacional complementaria- no han alcanzado los cuatro objetivos básicos de identidad, calidad, pertinencia y menor deserción, los cuales a su vez fungen como indicadores del derecho a la educación.

La asequibilidad es la dimensión del derecho a la educación que enfrenta enormes retos en cuanto a la obligatoriedad en este nivel. Aún se observa un déficit en materia de cobertura y distribución equitativa. Los indicadores educativos en nivel medio superior -el rezago, los porcentajes de reprobación y de deserción y la tasa de terminación-, determinan un marco de acción de las instancias estatales y gubernamentales para mejorar la eficiencia terminal y evitar el rezago, así como paliar las causas de la deserción con políticas públicas que reduzcan la desigualdad de oportunidades. Otro reto importante es la reducción de las brechas educativas, la transparencia en la asignación de los recursos públicos destinados a educación media superior, la profesionalización docente, la mejora de la propuesta 
curricular, metodológica y del equipamiento e infraestructura escolar. Por otra parte, es importante revisar las prácticas de evaluación con miras a un modelo más formativo y menos meritocrático que ha permeado todo el nivel medio superior en todas sus etapas: ingreso, permanencia y egreso.

En cuanto a otras dimensiones del derecho a la educación en este nivel -la accesibilidad, la aceptabilidad y la adaptabilidad- su análisis invita a una reflexión más profunda sobre las orientaciones y los objetivos de la política educativa actual. Los retos que persisten en materia de igualdad de oportunidades, calidad educativa e inclusión efectiva a pesar de las estrategias diseñadas por el Estado son algunos de los indicadores que conducen a la conclusión anterior. Asimismo, abre importantes cuestionamientos sobre los alcances y la falta de focalización de los programas sociales que podrían repercutir en mayor igualdad de oportunidades y, a su vez, en una mejoría del logro educativo en escala nacional.

\section{Referencias}

Aboites, H. (2012). El derecho a la educación en México. Revista Mexicana de Investigación Educativa, $17(53), 361-389$.

Amézquita, C. (2014). El derecho a la educación: Su valoración desde las vivencias situadas y diferenciadas de los y las estudiantes en el contexto escolar. Educación y Ciudad, 27, 99-107.

Andere, E. (2013). La escuela rota: sistema y política en contra del aprendizaje en México. Ciudad de México: Siglo XXI.

Bolívar, A. (2005). Equidad educativa y teorías de la justicia. REICE. Revista Iberoamericana sobre Calidad, Eficacia y Cambio en Educación, 3(2), 42-69.

Cesop. (2015). Evaluación del gasto educativo en México. Ciudad de México: Cámara de Diputados.

Chizzotti, A. (2016). Políticas públicas: direito de aprender e avaliação formativa. Revista Práxis Educativa, 11(3), 561-576.

COPEEMS. (2014). Reporte de la encuesta nacional de deserción en la educación media superior. Ciudad de México: SEP.

Didier, M. M. (2012). La exigibilidad judicial de los derechos sociales básicos: un imperativo del principio de igualdad. Persona y Derecho. Revista de Fundamentación de las Instituciones Jurídicas y de Derechos Humanos, 66(1), 81-107.

DOF. (2008). Acuerdo 444. Consultado el 30 de enero de 2017. Recuperado de http://www.sems.gob.mx/es_mx/sems/acuerdo_secretarial

DGAIR. (2000). La estructura del sistema educativo mexicano. Ciudad de México: SEP.

Hamui S. y Villa, L. (2010). ¿Continúa la reforma de la educación media? Observatorio Ciudadano de la Educación. Recuperado de http://www.observatorio.org.

Ibagón, N. J. (2015). La educación, un derecho que cuesta: dimensión fiscal y su relación con la política educativa en América Latina. Educación y Humanismo, 17(29), 29-37.

INEE. (2015). Panorama educativo de México 2014. Ciudad de México: INEE

Latapí, P. (1993). Reflexiones sobre la justicia en la educación. Revista Latinoamericana de Estudios Educativos, 23(2), 9-41.

Latapí, P. (2009). El derecho a la educación. Su alcance, exigibilidad y relevancia para la política educativa. Revista Mexicana de Investigación Educativa, 14(40), 255-287. 
Loyo, A. (2010). Política educativa y actores sociales. En A. Arnaut y A. Giorguli (Coords.), Los grandes problemas de México (pp. 185-208). Ciudad de México: COLMEX.

MAES. (2015). Historia. Recuperado de http://aspirantesexcluidos.blogspot.mx/p/historia.html.

Muñoz, C. y Núñez, M. (2004). Algunas consideraciones en torno al derecho a la educación y a sus beneficios socioeconómicos: El caso de México. En J. Auping (Coord.), El análisis económico de los derechos humanos (pp. 195-214). Ciudad de México: Universidad Iberoamericana.

OCDE. (2016a). Mexico: Overview of the education system (EAG 2016). Recuperado de http://gpseducation.oecd.org/CountryProfile?primaryCountry=MEX\&treshold=10\&topi $\mathrm{c}=\mathrm{EO}$

OCDE. (2016b). Panorama de la educación 2015. Nota de país: México. Recuperado de https://www.oecd.org/mexico/Education-at-a-glance-2015-Mexico-in-Spanish.pdf

OCDE. (2016c). PISA 2015. Resultados clave. Recuperado de https://www.oecd.org/pisa/pisa2015-results-in-focus-ESP.pdf

Ruiz, M. (2012). Derecho a la educación: política y configuración discursiva. Revista Mexicana de Investigación Educativa, 17(52), 39-64.

SEMS. (2013a). Estructura. Ciudad de México: SEP.

SEMS. (2013b). La educación media superior en el sistema educativo mexicano. Ciudad de México: SEP.

SEMS. (2013c). Principales cifras del sistema educativo nacional 2012-2013. Ciudad de México: SEP.

SNIE. (2013). Reporte de indicadores educativos. Ciudad de México: SEP.

Solís, P. (2010). La desigualdad de oportunidades y las brechas de escolaridad. En A. Arnaut y S. Giorguli (Coords.), Los grandes problemas de México (pp. 599-622). Ciudad de México: El Colegio de México.

SPEC. (2016). Planea. Publicación de resultados 2016. Ciudad de México: SEP.

Tomasevski, K. (2004). Indicadores del derecho a la educación. Revista Instituto Interamericano de Derechos Humanos, 40, 341-388.

Yanes, P. y Peralta, J. (2006). Las exclusiones de la educación básica y media superior en el D. F. Ciudad de México: Gobierno del Distrito Federal y Fondo de las Naciones Unidas para la Infancia.

Zorrilla, J. F. (2005). La importancia del sentido formativo del bachillerato. En E. Agüera, J. Vázquez y W. de Vries (Coords.), Retos y perspectivas de la educación superior (pp. 59-78). Ciudad de México: BUAP.

\section{Breve CV de las autoras}

\section{María Mercedes Ruiz Muñoz}

Académica de tiempo completo del Departamento de Educación de la Universidad Iberoamericana Ciudad de México. Doctora en ciencias con especialidad en Investigaciones Educativas por el DIE-Cinvestav. Integrante del Sistema Nacional de Investigadores, nivel III. Ha realizado estancias de investigación y de formación en reconocidas universidades de Estados Unidos, Brasil, Canadá y Argentina. La línea de investigación de sus trabajos se refiere al derecho a la educación y la justicia escolar. Entre sus distinciones destaca el Premio Pablo Latapí sobre Investigación Educativa. Actualmente coordina el Campo Estratégico de Acción en Modelos y Políticas Educativas 
del Sistema Universitario Jesuita. ORCID ID: 0000-0002-3759-4688. Email: mercedes.ruiz@ibero.mx

\section{Alejandra Luna Guzmán}

Estudiante de tiempo completo de la Maestría en Investigación y Desarrollo de la Educación de la Universidad Iberoamericana Ciudad de México. Becaria del programa de Ayudantías de Investigación como parte del proyecto La resignificación de la reforma educativa de 2013 adscrito al Instituto de Investigaciones para el Desarrollo de la Educación (Inide) de la misma universidad. Becaria de posgrado del Consejo Nacional de Ciencia y Tecnología (Conacyt). ORCID ID: 0000-0003-3574-8505. Email: alekalg73@gmail.com 